\title{
Wavelet Analysis for Classification of Multi-source PD Patterns
}

\author{
E. M. Lalitha and L, Satish \\ Dept. of Hith Woltage Ling. \\ Indian Institate of Science, Bangalore, Intia
}

\begin{abstract}
ABSTAACT
Multi-resolution signal decomposition (MSD) technique of wavelet transforms has interesting properties of capturing the embedded horizontal, vertical and diagonal variations within an image in a separable form. This feature was exploited to identify individual partial discharge (PD) sources present in multi-source $1 \mathrm{D}$ patterns, usually encountered during practical PD meastrrements. Employing the Daubechies wavelet, foatt res were extracted from the third level decomposed and reconstructed horizontal and vertical component images. These features were found to contain the necessary discriminating information corresponding to the individual $P D$ sources. Suitability of these extracted features for classification was further verifted using a radial basis function nemral network (NN). Successful tecognition was achieved, even when the constituent sources produced partialiy and fully overlapping patterns, thus demonstrating the applicability of the proposed novel approach for the task of multi-source PD classification,
\end{abstract}

\section{INTRODUCTION}

$\mathrm{D}$ LAGNOSTIC measurements are performed to assess the status of insulation systems in HV power apparatus. This has been a topic of continued interest in the past couple of decades. Presently there is a greater emphasis in the power industry and anongst utilities to perform on-site, and cven on-line diagnostic measurements [1]. This philosophy has been influenced primarily by the increasing use of digital hardware and computers (which afford making such measurements), followed by possibilities of post-processing the gathered data. Among the various diagnostic techniques, partial discharge ([D) measuroment is generally considored important, since it is nondestructive, non-intrusive and can qualitatively describe the overall integrity of the insulation system. Use of digital PD equipment is now more or less a standard, a fact recognized even by the forthcoming [EC Standard-60270 [2]. The data gathered from a digital pD detector is popularly referred to as a $\phi-q-r$ pattern, or PD fingerprint. These patterns contain certain characteristic features that are representative of a partictlar class, and this criterion has been used widely for recognition of the PD source [3].

In the opinion of the authors, there are basically two outstanding issues associated with the analysis of acquired PD data. The first onc is that of effectively storing, handling and subsequently processing the large mass of $P$ ro data to perform analysis. Such a situation can be visualized during PD monitoring of power apparatus during its service life. Additionally, it is also well known that insulation behavior is better understood and assessment of its status far more reliable, when l'v data gathered under sorwice life conditions, over large intervals of time, is available. Thus arises the necossity of accumulation and processing a large amount of data. $\Lambda$ possible solution addressing this issue was presented recently by the authors [4].

The second issue concerns processing the acquiled po data for identification of the PD sourcc. The last decade has seen much research in this arca. Various features have been extracted and classification was performed using different patten recognition methods, with varying degree of success. However, in a majority of these approaches, the pres ence of only onc l'D source being active at a given instant, is assumed implicitly.

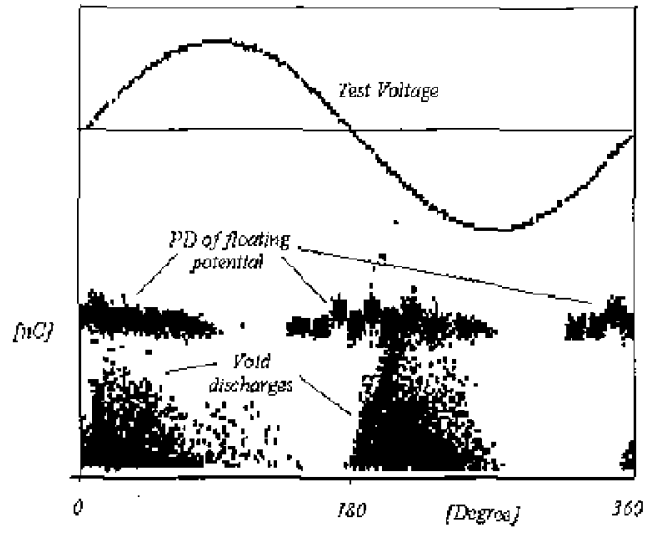

Figure 1. A multt-source PD patteril acquired from a generator stator winding $[6]$.

Unfortunately, the situation encountered duning practical PD measurements is far different, and most often, more than one source of $P D$ is active simultaneously; the sources could be intornal to the apparatus insulation or may be due to interference. In the latter case, there cxist 
a few mothods like gating/windowing, pulse discrimination and digitak filtering, that are employed to suppress its influence. When more than one PD source is simultaneously active, the resultant PD pattern obtained will in a broad sense be the sum of the patterns due to the individual sources. Stricly speaking, the pulse resolution time of the ID detector plays a crucial role in this situation. Given a short pulse resolution time, nost of the pulses will be well resolved, and then it would be fair enough to consider the resulting pattern to be a sum of individual po patterns. This has boon verified experimentally [5]. It generally is agreed that identification of the constituent sources in a $P D$ pattern with one or more sotrces producing ovarlapping patterns is quite involved. As an cxample, Figure 1 shows a ['0 pattern (drawn from [6]) due to singular floating potential and woid discharges in a stator winding. The fact that this task is still possible in the above figure is mainly because the pattern contributions due to individual sources ate as yet non-overlapping. The recognition task tends to become progressively difficult when the degree of overlap increases along both the magnitude and phase axes. Even for an expert, when presented with such overlapping patterts, it apparently becomes diffictult to discriminate and identify the presence of individual sources, despite having a knowledge of the shape of the PD patterns duce to individual sourocs. Since such situations are not uncommon during practical PD measurements, it will be worthwhile to explore newer methods capable of addressing such tasks. This preciscly is the aim of this paper.

In recent years, some prelininary investigations have been made for classification of multiple source PD patterns. A stochastic procedure based on mixed heibull functions applied to j'D pulse-height distributions was reported tn [7]. $\Lambda$ two-parameter Weibull function was observed to be sufficient to characterize the PD pattern of individual source, while a five-paraneter Woibull function was necessary for the mixed pattems, [inally, it was concluded that if one l'I phenomena completely or partially superimposes onto the other, identification and separation of the constituents would be virtually impossible [7]. The use of a notral network (NN) for separation of the constituent sources from a two-source PD pattern was cxamined [8]. It was concluded that the trained $N N$ yielded incorrect results when presented with $\mathrm{P} D$ patterns, for which it was not trained. Making a passing reference to this task, Pearson et $n$. used a NN and concluded that, if the contribution of one of the two sources considered is $>50 \%$, the $N$ decides on the source whose percontage in the mix is larger [9]. Such performances are, porkaps due to non-scparability of the features (corresponding to constituent sources) being fed to the NN. Separation of constituent P' sources from a two-soutce pattern was attempted by contour identification of the clarge and phase distributions [10]. If was concluded that the superposed patterns can be separated only if there was not much overlap of the two individual patterns. Use of statistical features also have been examined for this task [11] , but it was reported that the method is not fully suitable and the necessity for improved methods was highlighted. Thus, from an andysis of the literature, it cmerges that the multi-source PD pattern rocognition task (in its true sense) as yet is an umosolved issue, and hence the subject of this paper.

In this contribution, the authors address the task of multi-source ["] recognition, considering two soluces to be simultaneously active and producing partially or fully overlapping l'D patterns. A prior knowl- edge of PD patterns due to individual sources is the only information being assumed to be available. The novel use of wavelet transforms for this task is investigated, in particular multi-resolution signal decomposition (MSD). Results illustrate that even when the two sources produce complecly superposed lי pattens, it is still possible to separate the individual l'v sources, thus demonstrating the potential of this new method.

The paper is organized as follows. Section 2 deals with a short review of wavelet transforms and multi-resolution signal analysis concopts. The underlying principle of how this method can be adopted to the multi-source TD problem is discussed in Section 3 . A brief introduction to digital PD measurements and the pattern database used is presented in Section 4. Details of the proposed scheme, methot of feature extraction and a discussion of classification results are presented in Section 5 , folowed by conclusions and references.

\section{WAVELET TRANSFORM AND MSD}

The representation and analysis of digital data using wavelets has emerged as a very powertul and popular tool in recent years, with significant successes in areas stch as data compression, image and speech. processing, remote sensing, medical imaging and so on $[12,13]$. The wavelet transform, like the Fourier transform, decomposes a given signal into its frequency components, but differs in providirg a nonuniform division of the frequency domain. Unlike the Fourier transform, if provides a local representation in both time and frequency simultancously. This results from the fact that the analyzing or basis functions in the case of Fourier transiorm (namely sines and cosines) extend over infinite time, whereas they are compact functions of time in the case of wavelet transforms. Mathematically, the contiruous wavelet transform (Chr) of a function $f(t)$ with respect to a mother wavet $g(t)$ is defined as $[12,13]$

$$
\begin{aligned}
W_{s}[f(t)](a, b) & =W_{g} f(a, b) \\
& =|a|^{-0 . i} \iint(b) g^{*}\left(\frac{t-b}{a}\right)
\end{aligned}
$$

where $a$ is the scalc factor (real, $a \neq 0), b$ the translation parameter $r_{i}$ and $*$ is the conjugation operator. A mathematical restriction on the choice of $g(t)$, called the admissibility condition, exists. Some exomplos of popularly used mother wavelet functions are Morlet, Daubechies, Haar, Coitman, etc. The choice of the mother wavelet depends on the application and no general rule exists for its selection. The function $W_{g} f(a, b)$ gives an indication of the contribution of the signal around time $b$ and scale a i.e. signal information at different resolutions. IIowever CWT is computationally cxpensive and also generates much redundant data, To circumvent these drawbacks, an effective implementation applicable to discrete signals, called the discrete wavelet transform (Dw1') was formulated using suitable filters, which sotisfy cottain constraints. An clegant procodure called the multi-resolution signal decomposition (MSI) technique [12] is implemented through this method, which is tho primary reason for the widespread use of wavelets.

In DwT the signal is passed through a low-pass filter $\mathbf{G}_{A}$ (sce Figure 2(a) $)_{f}$ called the scaling function, and a high-pass filter $\mathbf{H}_{A}$, called 


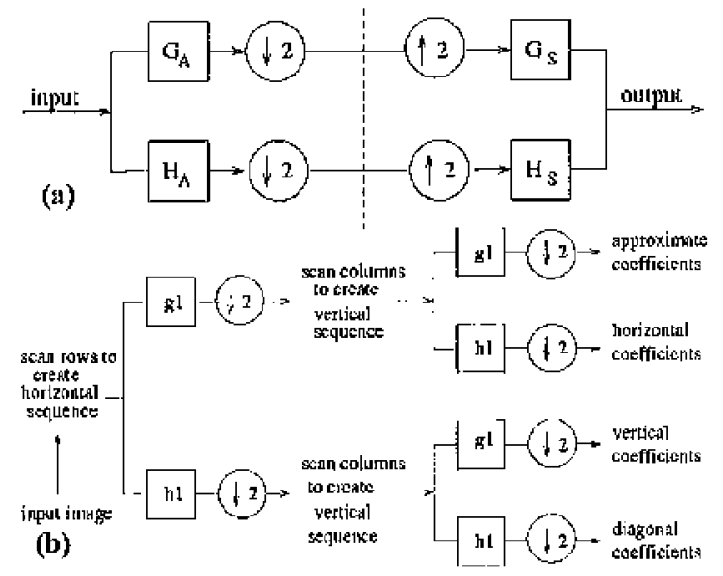

Figure 2. Block diagrams depicting the concepts of MSo (a) single-stage analysis and synthesis (b) single-stage analysis of an image. $\{\bar{a}\}$

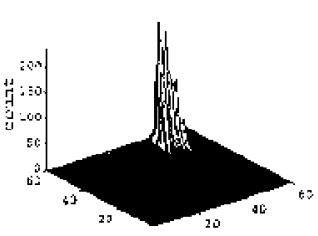

(h)

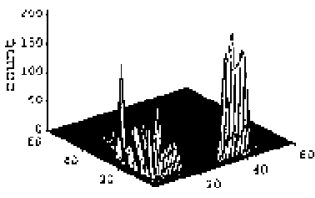

(c)

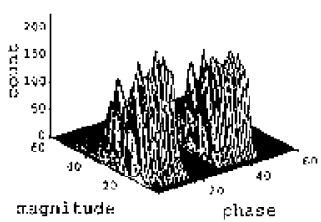

(a)

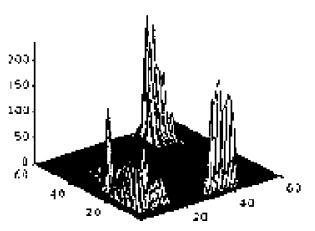

lok

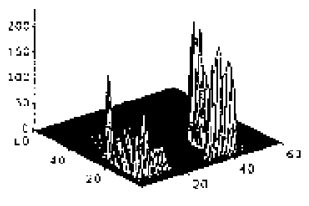

[F]

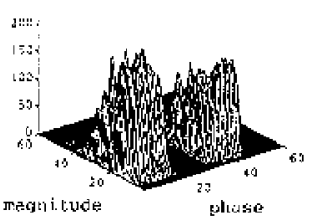

Figure 3. ro patterns of individual and superposed sources (a) single point corona in air, (b) surface discharge, (c) cavity discharge, (d) superposed $(a)$ and $(b)_{r}$ (e) superposed (a) and (b) with magnitude shift in $\{a)_{h}(f)$ superposed (b) and $(c)$.

the mother wavelet, and then cach output is downsampled by a factor of two. The output of $\mathbf{G}_{A}$ (approximate coelficients) are the highscale (low-frequemcy) components and output of $\mathbf{H}_{A}$ (detailed coefficients) are the low-scate (high-frequency) components. The detailed cocfficients represent the signal's characteristics and energy at higher frequencies, while the approximate coefficients are a blurted version of the original signal. The deconposition process (analysis stage) can be iterated with successive approximate signal components being decomposed in turn, so that the signal is further divided into many levels of lower resolution components. Thus, it gives information of the signal at different resolutions and this MSD popularized the concept of wavelet analysis. Analogous to the decomposition scheme, the signal coefficients can be reconstructed (synthesis stage) using another set of filters $\left(\mathbf{G}_{s}, \mathrm{H}_{s}\right)$ to give the extent of signal prosent at different scales. The analysis and synthesis filter pairs are inter-related and referred to as guadrature mirror filters, Imposition of certain constraints on these sets of filters ensures a perfect reconstriction. In other words, summing all the reconstructed signal components (obtained at different lovels of dccomposition) yields the original signal.

For a 2D signal, say an image, the rows and columns arc considered separately for decomposition. Figure 2(b) gives the detailed block diagran of single stage decomposition of an image. The horizontal and vertical sequences are separately filtered and subsampled. When the scquences are processed as shown in the Figure, the output of decomposition at crery level yields four sub-images, namely onc approximate and three detailed coefficients. In the reconstruction process, these coefficients are agair upsampled and filtered; for details see $[12,13]$. The reconstructed component images are referred to as approximate $(A)$ and detailed hotizontal ( $H$ ), vertical (V), and diagonal (D) images. A blurred version of the input image is represented by $A$, whereas $H$, $V$ and $D$ characterize the horizontal, vertical and diagonal variations of the original image respectively. Here also, a sum of all the reconstructed images (corresponding to each level of decomposition) yields the original inagc.

\section{MULTI-SOURCE PD PROBLEM}

\subsection{AN EXAMPLE}

An example highlighting the difficulty encountered in separation of individual 50 sources from a multi-source lo pattern is illustrated in Figure 3. Three po sources viz, single point corona in air, surface discharge and cavity discharge are considered and shown in Figures 3(a) (b) and (c), respectively. A $3 \mathrm{D}$ view of the patterns is given for purposes of clarity. Figures $3(\mathrm{~d})$, (c) and (f) are the two-source Iro patterns obtaited by digitally supurposing two of the single source PD patterns. In Figure $3(d)$, which is the result of adding Figures 3(a) and (b), it is scen that the PD pulse clusters of the two sources are non-overlapping. Hence, an expert with some knowledge of the shape of PD patterns from individual sources, can identify the presence of sources by analyzing the phase and magnitude positions of the clusters, together with the extent of spread along each axcs.

Usually in practical 'p measurements, pulse cluster's of individual 12D sources are either partially or completely overlapping. Under such conditions, it is almost impossible to identify, by visual inspection, the presence of different sources. The partial and complete stuperimposed cases are shown in Figures $3(e)$ and (f) respectively. Figure $3(e)$ is the stperposition of Figure 3(a) and (b), with a magnitude shift intensionally incorporated into Figure 3(a). The individual pulse cluster information being not clear in Figure 3(e) leads to some unceraingy and thereby causes an ambiguity in recognition. In some cases, phase and nagnitude spreads, and also the position of pulse clusters may be similar, leading to a complete overlap. This is ovident in Figure $3(\mathrm{f})$, which is obtained by adding Figures 3(b) and (c). When Figure $3(f)$ is exnmined, even with prior information that two sources are present, it is very difficult to discriminate the presence of the sources. The principle of how 
wavelet transforms can be used effectively to resolwe such recognition tasks, is discussed next.

\subsection{UNDERLYING PRINCIPLE}

Use of wavelets in andyzing an input signal or image by decomposition of the input and subsequont reconstruction of only those portions that are of interest, assists in improved analysis, since each portion can be analyzod sepantely. Figure 4(a) shows a completely overlapping multi-source l's pattern (sulface discharges and cavity discharges) plotted as a 64 gray-scale inage. A three level inso tusing the Daubechies wavelet with 56 cocficients was implemented on this image. A reconstruction at the third level yielded four sub-images, namely, the approximate (A), horizontal ( $\mathrm{H}$ ), vertical (V) and diagonal (D) images, and only these are shown in Figures $4(b)$ to (e).
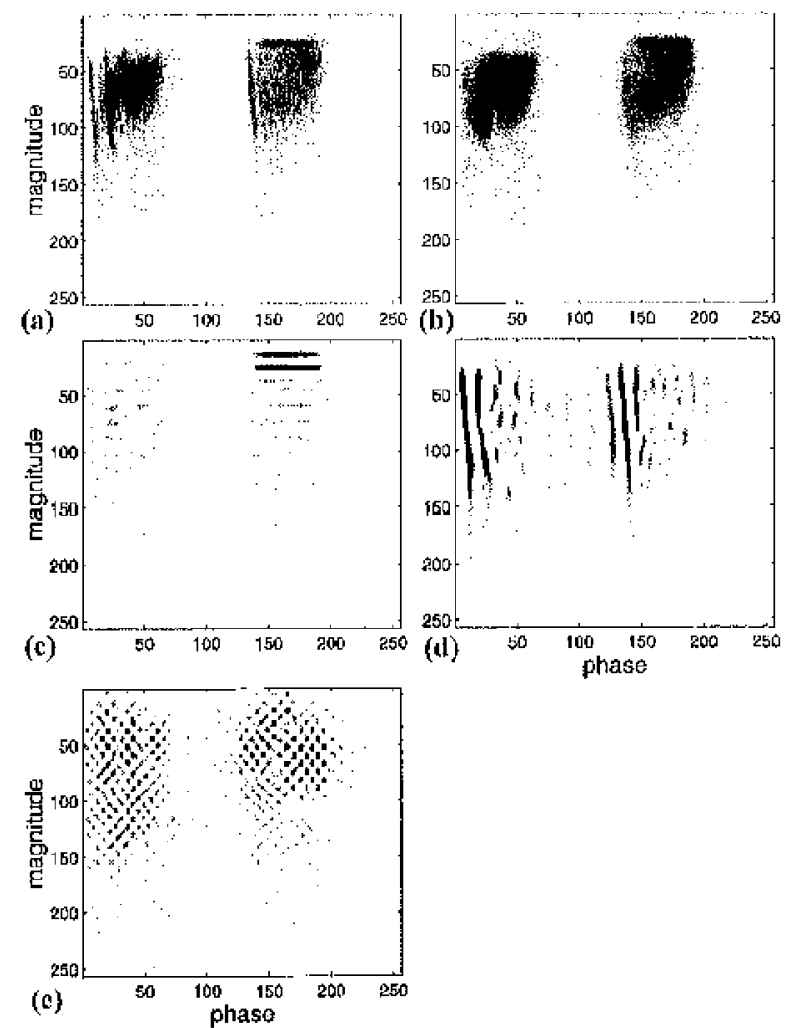

Figure 4. Original and lhird-level reconstructed images of the superposed PJ pattern in Figure 3(f), (a) original (b) approximate (c) horizontal (d) veitical (e) diagonal.

Figure $4(b)$, the approximate image $(A)$, is a smoothed version of the original image. On visut inspertion, it is crident llat this image contains no more additional information when comparcd to the original inage. Figure 4(c) represonts the horizontal variations and Higure $4(d)$, the vertical variations enbedded in the original. Figure 4 (e) shows diagonal variations present in the original, and similar to Figure 4 (b), does not seem to contain any specific intormation pertaining to the two solurces. After a considerable amount of study of all the $\mathrm{A}, \mathrm{V}, \mathrm{H}$ and $D$ inuges, obtained from processing different single and multi-source patterns, it energes that only the II and $V$ images contained relevant information, that perhaps could aid in separation of the constituent sources present in the input. The basis of choosing II and $V$ image componetits from among the four sub-images was by visual inspection.

$A$ decision regarding the number of levels of decomposition and the choice of mother wavelet coefficients was made after an extensive examination of all the reconstructed image components at different levels, arising due to different multi-source pro patterns. The studies revealed that a 3 -level decomposition-reconstruction using the Daubechics wavelet with 56 coeficients was found to be adequate. Further, restricting analysis to only the third lovel images turned out to bo sufficient for the purposes of the underlying task. Lastly the ability to provide a separation of features present in the original image into cach one of the decomposed components is a fundamental property afforded by the multi-resolution analysis [12]. Fxplanation on how these $\mathrm{H}$ and $\mathrm{V}$ images represent features that can lead to discrimination and classification of the individual plo source is explained below with an example.

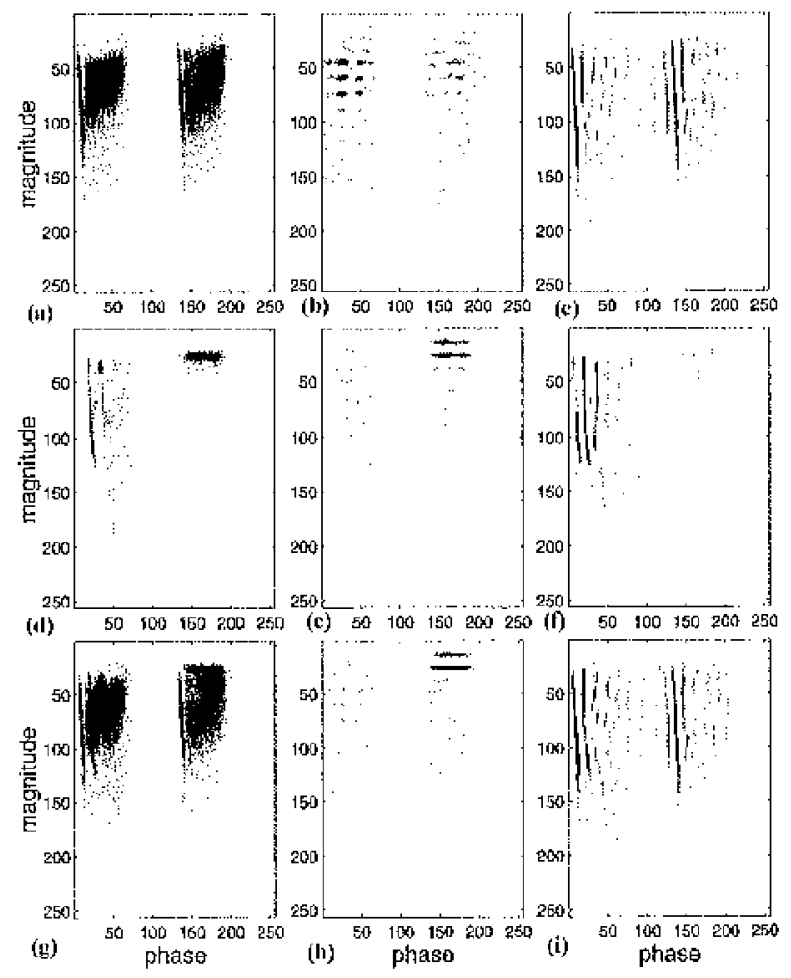

Figure 5. H and $V$ images of individual and superposed rD patterns (a) cavity discharge, (b) $\mathrm{H}$ image of (a), (c) Y image of (a), (d) surface discharge, (c) H image of (d), (f) V mage of (d), (g) (cawily + surface) discharge, (h) $\mathrm{H}$ image of (g), (i) $V$ image of (g).

Figures $5(a)$ and (d) are the patterns due to individual sontes (cavity discharges and surface dischatges respectively) and Figure $5(g)$ that due to superposition of these individual sources. [igures $5(\mathrm{~b})$, (e), and (h) are the third level decomposed and reconstructed $\mathrm{H}$ images, and Figures $5(\mathrm{c})$, ( $\mathrm{f}$ ) and (i) are the third level decomposed and reconstructed $V$ images of those shown in Figure 5(a), (d) and (g) respectively. Fxplamation of the obserwations is made by considering the first and sccond half cycles of the sine-wave separately (pixels 1 to 128 and 129 to 256 
in the horizontal direction represent the first and second half cycles respectively).

In Figure $5(\mathrm{~b})$, the $\mathrm{H}$ components of cavity discharges are not so prominent in both half cycles. In contrast, in Figure 5(c), the presence of dark lines in the horizontal direction clearly indicates that the II component of surface discharges is very prominent in the second half cycle. The H component of the superposed image should thus reflect these properties. Inspection of rigure $5(h)$ reveals that the II components arc less significent in the first half cycle, but are very prominent in the second half cycle, thus tevealing the possible presence of surface discharges. Next, consider the $V$ images of the individual and the superposed images in Figure $5(c)$, (f) and (i) respectiwely. It is seen that in. [igure $5(\mathrm{c})$, the $\mathrm{V}$ component is prominent (indicated by dark vertical lines) in both pulse clusters. In Higure $5(\mathrm{f})$, the $V$ component is prominont in the first half cycle only. This is indicated by dar'k vertical lines in the first half cycle. The $V$ intage of the superposed case (Figure $5(i)$ ) shows the presence of prominent $V$ components in both the half cycles, indicating the possible presence of cavity discharges. The proposition of only considering the $H$ and $V$ images was confirmed, when similar observations were made, whilc examining several examples of multisource $P D$ patterns along with their single source pattorns.

To summarize, visual inspection of the $\mathrm{H}$ and $\mathrm{V}$ images of the multisource image (Figure 5 (h) and (i)) reveals that the salient features which are present in the individual sources, also are present in them, and mos: importantly, are available in a scparable form. The fact that such a separation was possible by this procedure is the underlying principle that was exploited. Additionally, it was also observed that the phase and magnitude positions of the individual sources remain unaltered in Figurcs 5(h) and (i) respectively, although it is not readily distinguishable in the original image. Very similar olservations were scen in the other two cases viz partial and non-overlapping situations. These observations, in genoral, were found to exist in all the multi-source PD patterns investigated. Salient features are dofined and extracted from the $\mathrm{H}$ and $V$ components for classification of individual PD sources present in the two-source PD pattern. In this manner, a simple and workable solution is proposed for identifying the individual PD sources present in a given I') pattern.

\section{DETAILS OF PD PATTERNS}

\subsection{BRIEF INTRODUCTION TO PD}

$\mathrm{PD}$ is a localized broakdown event accurring due to minute defects in insulation structures. These defects ate inevitable during manufacture or could develop during the service life of the apparatus, in spite of the best efforts and procautions. PD tsually is a pulsive eqent of very short rise time and relatively longer fall time (at defect sites), and these values depend on the type of insulation, Quantifying of $P D$ in terms of apparent charge is very popular and correlates well to the overall insulation degradation [1].

The digital ('I) detector is actually an extension of the analog counter ${ }^{\sim}$ part. It involwes digital acquisition of all the individual quasi-integrated pulses (to the extent supported by the hardware) and quantifying each of them by their apparent charge magnitude $(q)$, the corresponding phase angle $(\phi)$ at which they occur, and their number densities $(n)$ over an interval of time. In other words, the phase and charge axcs are divided into bins and the pulses are sorted into these bins, finally yielding the $\phi-t r-n$ paltern. As can be seen from the Higures, $\Gamma D$ pulses tend to occul' in clusters and, in general, rD patterns from a particular source occupy specific phase positions and spreads. This information is vital for recognition purposes.

\subsection{PD PATTERN DATABASE}

Three types of PD patterns were considered in this study. They are single point corona, cavity discharge and surface discharge. It is well known that a good database is an essential pre-requisite for developing a robust pattern classification system. Keeping this requirement in mind, cxperimentally gathered pls pattern data from two different measuring systems and recorded under different experimental conditions (in laboratories abroad, see acknowledgments) were pooled to form the database. Simple defect models were used in the experiments. Thus, the database included $\mathrm{PD}$ patterns acquired from two different detectors (namely a Hacfely PD measuring system, TF.AS 570, and a lettex PD measuring system, Type 9126), recorded at different times, at different voltage levels, but arising basically due to a similar IDD source. The PD patterns are stored as $256 \times 256$ and $200 \times 200$ matrices respectively by the two detectors. In this work, all patterns were resized to $256 \times 256$ and nomalized, prior to processing. The original single source pattens were $\sim 15$ for each source. It is believed that a wider variety has been introduced by using such a database.

Using these single source patterns, muiti-source ID patterns were artificially generated by digital superposition, followed by normalization. To generate partial and completely overlapping PD patterns, the individual pulse clusters of one source were shifted, if necessary, with due care exercised to incorporate realistic phase and magnitude positions (usually observed during practical pD measurements). The shift in magnitude direction was along the entire magnitude axis, while the phase shift was of the order of $上 20^{\circ}$. In this way nulti-source $\mathrm{rD}$ patterns were generated. The multi-source ID patterns considered for classification ate:
1. Case A: single point coront and carity discharge.
2. Case B: single point corona and surface discharge.
3. Case $\mathrm{C}$ cavity discharge and surface discharges.

Each of these patterns were decomposed to three levels, employing the Daubechies wavelet with 56 coefficients.

\section{RESULTS AND DISCUSSION}

\subsection{FEATURE EXTRACTION}

As explained in Section 3, the third level reconstructed $\mathrm{H}$ and $\mathrm{V}$ images (having a size of $250 \times 256$ ) of the superposed Pr pattern represent salient features pertaining to the individual sources in a separable form, and also preserve the phase and the magnitude positions of the individual PD pulse clusters and their phase spreads. Therefore, for classification, these II and V images must be represented suitably. Formulation of a stitable feature vector was carried out by aweraging the $\mathrm{H}$ and $\mathrm{V}$ images, both in the magnitude and phase directions. Avcraging in the phase direction retains the magnitude information and 
averaging in the magnitude direction retains information in the phase direction. The feature extraction procedure is summarized below and was performed for both the $H$ and $V$ inages.

Magnitude averaging: Image pixels are averaged viewing from the phase axis. This averages magnitude information and retains phase information, which is vital for classification. With a vicw to preserve salient features, and at the same time, minimize the lengths of these vectors ( $1 \times 256)$, different reductions were examined, and it was found that a length of fifly $(1 \times 50)$ was suitable, both in torms of achieving a reduction and for recognition purposes. The nearest-neighbor interpolation method (MATAB image processing toolbox) was ut]lyed for converting the $(1 \times 256)$ vector to a $(1 \times 50)$ vector.

Thase averaging: Averaging was performed viewing the image from the magnitude axis, i.e, phase values were averaged. These vectors contain the magnitude information only, which is important for classification, because in some cases, despite superposition of pulse clusters along phase axis, they are separable when vicwed along the magnitude axis. These vectors were lesized to a length of twenty five ( $1 \times 25)$ for the same reason stated above.

The final feature vector' was formulated by concatenating the averaged phase and magnitude vectors as follows: Let $I_{5,0}$ and $V_{5,1}$ be the magnitude averaged vectors, and $I_{25}$ and $V_{25}$ be the phase averaged vectors, then feature vector is $\left[H_{50} V_{50} H_{25} V_{25}\right.$ ], which is of length 150.
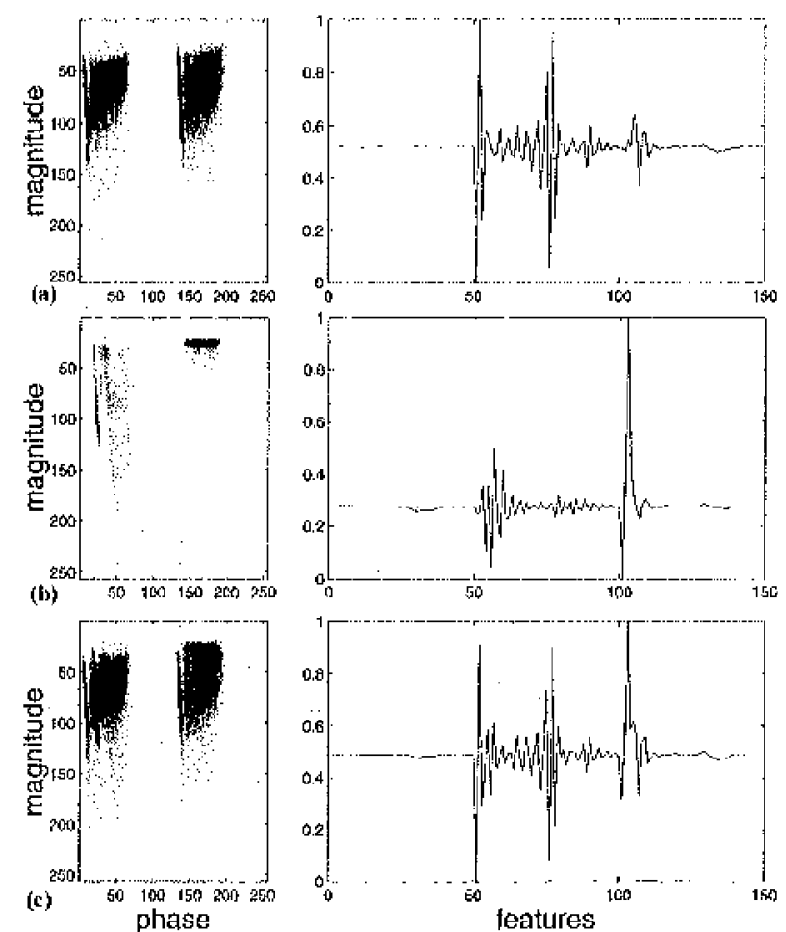

Figure 6. licalure wectors extracted for jndividual and superposed PT] images in Figure 5(a), (d) and (g) respectively. (a) cavity dischargc, (b) suiface discharge, (c) (cavity + surlace) discharge.

As an example, consider Figures 6(a) and (b) which shows the feature vectors for cavity discharge and surface discharge patterns (shown in

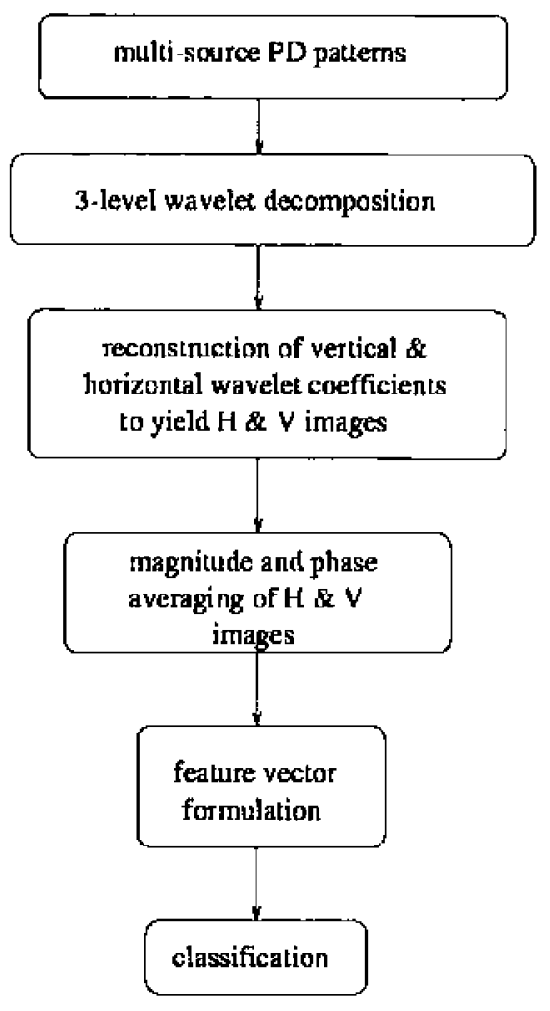

Figure 7. Scheme adopted for multi-sourec pD classification.

Figure 5(a) and (d)) respectively. Figure 6(c) is the foature vector corresponding to the multi-source PD pattern (cavity and surtace discharge) and shown in Figure 5(g). Figure 6(c) clearly indicates the separable form of the salient features present in the individual sources. Such a trend was observed in all the multi-source PD pattems examined. Next, these features are to be tested for their classification abilities. Figure 7 is a block diagram outlining the scheme of action.

\subsection{CLASSIFICATION}

In this work, a neural network (NN) was utilized solely to illustrate the classification abilities of the features extracted. A NN is reported to be suitable for clissification purposes due to its learning and generalization capabilities: In this work, the radial basis function NN was used. The advantage of this archilecture is its evolving structure, in contrast to the backpropagation algorithm, where architecture a priori has to be fixed by trial and error. Tearnitg in a radial basis function network is uquivalent to finding a surface in a multi-dimensional space that proFides a best fit to training data and gencralization is equiwalent to use of this multi-dimensional surface to interpolate the tost data. It uses the Euclidean norm for the distance measure, instead of derivatives, as in backpropagation algorithm, and hence has faster convergence [14].

The number of input layer netrots were 150 (equal to length of the feature vector) and the output layer had three neurons (equal to the mumber of multi-source patterns considered). The training-set had 60 patterns, 20 for ach type of two-source patterns considered. The convergence is considerced to be achieved, when the overall sum-squared 
Table 1. Classification ressults: the number of tost patterns $N$ and the number of correctly dassified patterns $l^{\prime}$.

\begin{tabular}{|c|c|c|}
\hline Case & $N$ & $P$ \\
\hline $\bar{h}$ & 150 & 1.50 \\
\hline D & 75 & and \\
\hline C & 90 & $\$ 1$ \\
\hline
\end{tabular}

error of the network was 0.001. This is the cumulative evron for one cpoch i.e. after all the training patterns are fed once. The number of cpochs required for the convergence was observed to be 60 , during difforent independent training sessions.
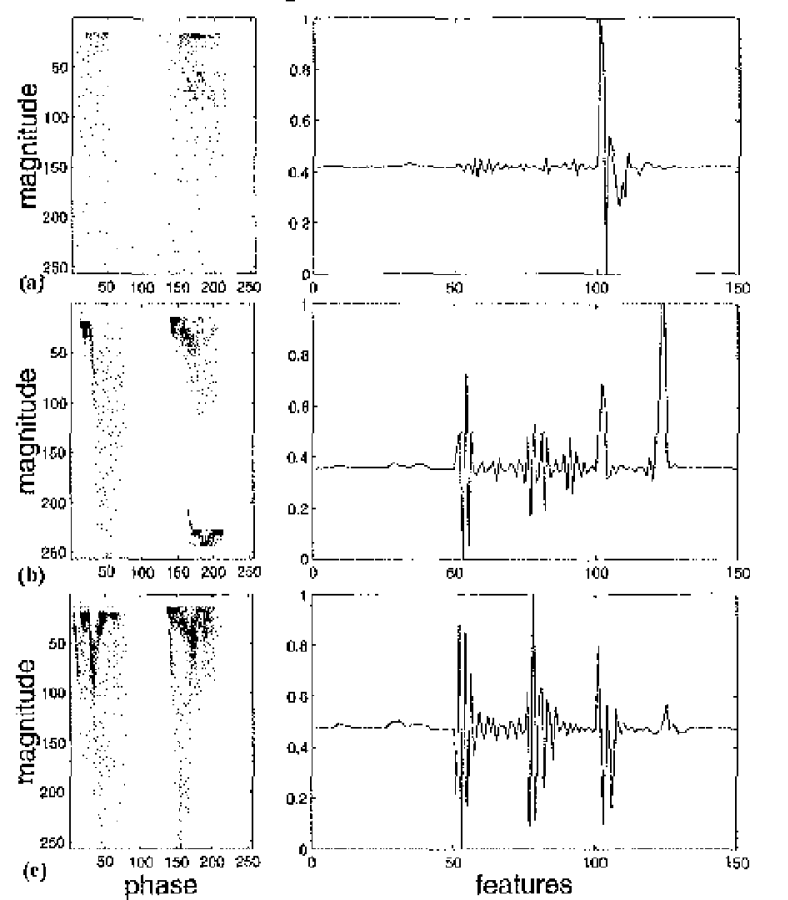

Figure 8. Example paltems of cases $\Lambda, B$ and $C$ that were correctly classiffed, allong with their feature vectors,

To tost the robustness of the foatules extracted, a certail amount of noise $(\sim 30 \%)$ and shift (as mentioned in Section 4 ), were added to the existing patterns and feature extraction was performed. These now features were then tested for classification, using the converged NN. Nearly, 100 patterns for cach type of tho multi-source J'D (and those not included in the training-set) were tested. A majority of these testing patterns were either partially or fully overlapping Po patterns. When the output layer neurons yielded a value $>0.6$, it was considered as classified into that aliss. In all, correct classification was obtained for $>88 \%$ of the patterns. As an example. Figure 8 illustrates multisource $\mathrm{PD}$ patterns (one ach from case $\mathrm{A}, \mathrm{B}$ and $\mathrm{C}$ respectively), which have been correctly classifict by the traned NN. Also showrt are the corresponding feature vectors. 'The results are summarized in Table 1.

A few instances of misclassifications were also obsored when the prominent features in II or V components in one of the sources were cxactly sinilar in size and plysical location of the second source, to which the first source was being added. Except for a few such extreme cases, more or less good recognition capabilities werc achieved, thus, demonstrating the potential of this new method. A najor contribution to the misclasfication were these multi-source patterns possessing ${ }^{2} \mathrm{X}$ cessive phase and magnitude shifts, for which the NR was not traincd for.

Since, the primary aim was to demonstrate the principle of this method, to begin with, only thee l'D sources were examined. However, multi-source l'o patterns gencrated from different individual sources, remain to be investigated, which will throw more light on the potential of this approach. To the question, as to how would the method behave, if more than two $P(\theta)$ sources are present, a speculative answer would be that the feature vector will have to be more carctully formulated. so that if could capture saithfully the salient foutures of the sources present. Also, it is very likely that when more sources are present, the weaker ones will got swamped by the more powertul ones. This is a matter of concern and further research is necessany to find appropriate answers. However, when two sources are present, the fature vector is robust enough to take carc of these artifacts.

\section{CONCLUSIONS}

$\mathrm{T}$ THF multi-source PD recognition problem, usually encountered during practical PD measurements, is as yet an unresolved task, except in a few cases where the individual po sources produce nonoverlapping patterns. In this contribution, the novel use of MSD (a kind wavelet of transform) has been demonstrated to be suitable for this task, even in the presence of fully overlapping p'o patterns. Employing the Daubedies wavelet, the multi-source l'D pattern, treated as an image, was subjected to a three level decomposition and reconstruction process. The third level reconstructed horizontal (H) and vertical (V) component images were observed to contain the characteristic pattern features corresponding to the individual sources, and most importantly, in a separable form. Using these $\mathrm{H}$ and $\mathrm{V}$ images, suitable fentures were extracted, which were then tested for their classification abilities using the radial basis function NN. Results analyzed (after $>300$ partinlly and fully overlapping patterns wore tested) seems to indicate the potential of this approach for this task. Iowever, further study with different and actual multi-source l'D pattorns should be performed to reveal truc capabilities and drawbacks, if any, of the proposed method.

\section{ACKNOWLEDGMENT}

The atthors wish to express their thanks to E. Gulski of 'I'U Delft, The Netherlands, for supplying some $\mathrm{PD}$ patterns that were used in an carlier joint contribution. Thanks are also due to Tettex Instruments AG, Dcitikon, Swit/crland, who have been kind to permit the use of some $P D$ patterns, recorded by the second author some years earlicr.

\section{REFERENCES}

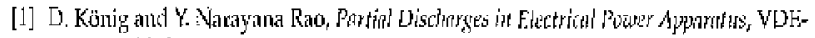
verlas publishers. 1993

[2] IJC Standare 601270, Parlial Discharge Masurements, Committee Draft, IEC TC 42, WG 11,39 IYVD, Zatengl, Sipt. 1945.

[3] E. Gulski anda F. H. KIenger "Computer-aided Recognition of Discharge Sources", TEFE Transactions on Flectrical Insulation, Vhe, 27, No. 1, pp. 82-92, Fob. 1992.

[4] F. M. Lalitha and L. Satish, "Fractal Jmage Compression Technoque for Classitica-

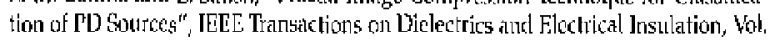
5. No. 4, pp. 550-557, Aug $199 \%$. 
[5] June-HoLes, Doo-Sumg Shin and 'I' Okimoto, "Analysis of Parlial Discharge Signals

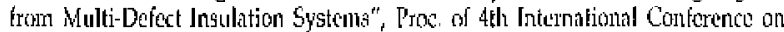
Properties and Applications of Diclect tic Materials, May 25-30, 1997, Scoul, Koreat.

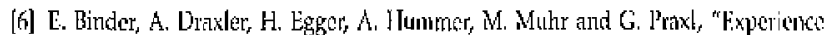
with on-line and off-line l'D menstuentents of generator's", CIGRE Session 1998, Paris, paper 15. 106.

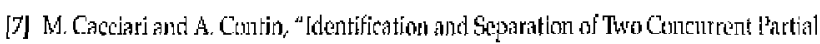
Discharge Phenoment", Conference on Hoctrical Insulation and Dielectric l'tenonena, San Fransisco, OcE. 20-23, 1996, Pp. 476-479.

[8] ] Junc-Ho Lee, Doo-5ung Shin and Thtshuki Okamotn, "Analysis of Partial Disclarge Signals from Multi-Defect Insulating Systens", Froc of 5th Int. Cont. mo Properties

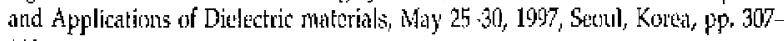
310 .

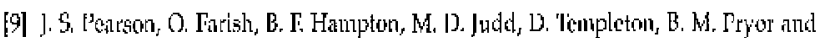

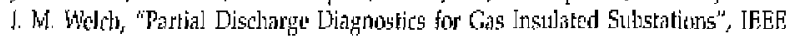

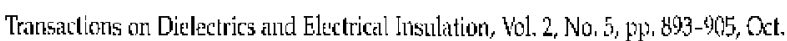
1995.

[10] C. Cachin and II. J. Wiresmann, "PD Recognition with Knowltedge-based [2thessing and Neural Networks", IEEE "Iransictons on Dielectics and Jectrical [nsulation, Vol. 2, No. 4, pp. 578-589, Aug. 1995

[11] A. Krivda and S. Halen, "[kecognition of I'U Pattel the in Generatos", I'roc, of 5 th Int, Conf. on l'topertics and Applications of Dielectric Malerials, May 2,-30, 1997, Scoul, Korea, pe. $216-211$

[12] Randy K. Koung, Waoplet dicdry aud applications, Kluwer Academic publishers, 1993.

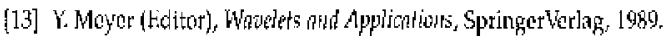

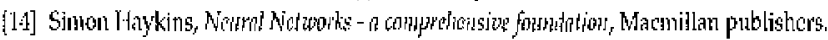
Manuscipt was rocelved on 12 Docember 1998 , in revised form 19 Novenbel 1999. 